\title{
Anti-Obesity Potential of Heritiera Fomes Leaves Methanolic Extract in a Preclinical Model
}

\author{
*M.Salim Mirza ${ }^{1}$, Sayed Ayaz Ali ${ }^{2}$, Indrajeet Sanghvi ${ }^{3}$ \\ 1. Research Scholar, Faculty of Pharmacy, Pacific University, Udaipur (Rajasthan, India) \\ 2. Associate Professor, Dept.of Pharmacology,Y.B.Chavan College of Pharmacy,Aurangabad(M.S.,India) \\ 3. Dean, Faculty of Pharmacy, Pacific University, Udaipur (Rajasthan, India)
}

\begin{abstract}
Objective: This study was undertaken to evaluate the anti-obesity potential of the methanolic extract of leaves of Heritiera fomes. (MEHF) in high fat cafeteria diet (HFCD) fed obese rats.

Methods: Wistar strain of albino rats were divided into six groups comprising of six rats each. Group I served as normal control fed with normal pellet chow, group II served as disease control fed with high fat cafeteria diet, group III, IV and V animals, received MEHF at a dose level of 100, 200 and 400mg/kg b.w along with HFCD for 40 days, while, group VI served as standard drug control, which received Orlistat at a dosage of $50 \mathrm{mg} / \mathrm{kg}$ b.w along with HFCD.

Results: Administration of HFCD for 40 successive days to experimental rats significantly increased the body weight, organ and fat pad weights, serum total cholesterol, LDL cholesterol, VLDL cholesterol, triglycerides and glucose levels; and decreased HDL cholesterol as compared to normal control. While treatment with MEHF showed a significant reduction in the body weight gain, organ weight of the liver, kidney, spleen, weight of fat pads and the levels of serum triglycerides, total cholesterol, LDL cholesterol, VLDL cholesterol, glucose and increase in HDL cholesterol in a dose dependent manner. Further, the levels of liver markers such as aspartate transaminase (AST), alanine transaminase (ALT) and alkaline phosphatase (ALP), which were found to be elevated in the serum of obese rats, also resumed to normal on treatment with different concentrations of MEHF. Moreover, the consumption of MEHF reduced oxidative stress by enhancing the levels of glutathione $(G S H)$, glutathione peroxidase (GPx), super oxide dismutase (SOD) and catalase in the hepatic tissue of rats with HFCD induced obesity.

Conclusion: These results demonstrate clearly that repeated oral administration of Heritiera fomes methanolic extract can evoke a potent anti-obesity activity.

Keywords: High Fat Cafeteria diet, Obesity, Heritiera fomes, Orlistat.
\end{abstract}

\section{Introduction}

Obesity and overweight, the most common nutritional problems resulting mainly from an energy imbalance caused by an increased ratio of caloric intake to energy expenditure represent rapidly growing threats to the health of population worldwide. The International Obesity Task Force estimates that more than 300 million individuals worldwide are obese with body mass index (BMI) $\geq 30 \mathrm{~kg} / \mathrm{m}^{2}$ and 800 million are overweight (BMI between 25 and $29.9 \mathrm{~kg} / \mathrm{m}^{2}$ ) [1]. Due to the rising trend in obesity prevalence, this figure could double by the year 2025, if no action is taken against this threat. Obesity decreases the quality of life and life expectancy and is also a strong risk factor for diseases such as type 2 diabetes, heart disease, stroke, certain types of cancers, osteoarthritis, liver disease, urinary incontinence, sleep apnea, and depression [2]. Left unabated, the increasing rates of obesity in the world will place a severe burden on national healthcare systems.

Despite advances in understanding its pathogenesis, current pharmacotherapy for obesity remains limited both in the degree of achievable weight loss and the safety/tolerability of the drugs. Thus, discovery of new targets and therapeutic agents is a focal point for combating this epidemic. A large section of world's population relies on traditional remedies to treat various diseases. Medicinal herb is an indispensable part of traditional medicine practiced all over the world due to its efficacy, low costs, easy access, ancestral experience and lack of side effects [3]. Hence, the present study was carried out with an objective to investigate the antiobesity effect of methanolic extract of Heritiera fomes. (MEHF) in rats fed with high fat cafeteria diet. Heritiera fomes belonging to the family Sterculiaceae is a well-known plant in Indian traditional folklore medicine, $\mathrm{H}$. fomes leaves, roots, and stems are used by rural people for the treatment of gastrointestinal disorders, skin diseases, and hepatic disorders. Bark is taken for diabetes and goiter in rural areas. This plant is also used as herbal medicine to cure pain and fever locally. It has been evaluated for Antidiabetic [4], antioxidant potential, antibacterial activity [5], anti-nociceptive, antioxidant and analgesic activity [6] and anticancer activity [7].In the present study attempt was made to evaluate antiobesity activity of MEHF in high fat cafeteria diet induced obese rats, so as to provide scientific evidence for this "Mangroove" drug. 


\section{Materials And Methods}

Preparation of Leaves Methanolic Extract of Heritiera fomes (MEHF)

Sun dried leaves of Heritiera fomes were stored at ambient temperature. Leaves were ground continually in a mechanical grinder and sieved until all raw materials had a granulometry of $\leq 0.5 \mathrm{~mm}$. The granulated powder was extracted with methanol by using Soxhlet apparatus. The extract was filtered and the filtrate was evaporated in a water bath till it reaches a thick consistency. The extract was stored in refrigerator till further use.

\section{Experimental Animals}

Healthy Wistar strain of Albino rats (6-7 weeks old) of both sexes weighing 100-150g obtained from Tamil Nadu Veterinary College, Chennai, India, and used for the present study. They were housed in polypropylene cages and maintained under controlled environment $\left(24^{\circ} \pm 1^{\circ} \mathrm{C}\right.$ temperature, $55-65 \%$ relative humidity, $12 \mathrm{hr}$ light/dark cycle) with free access to standard animal pellet diet (Sai Durga Feeds, Bangalore, India) and water. They were acclimatized to the laboratory conditions for a week before experiments. The animal experiments were conducted as per the ethical guidelines of CPCSEA after obtaining necessary clearance from the Institutional Animals Ethics Committee

\section{Induction of Obesity by Feeding High Fat Cafeteria Diet}

The cafeteria diet [8] consisted of 3 diets (condensed milk, 40g + bread, 40g), (chocolate, $15 \mathrm{~g}+$ biscuits, $30 \mathrm{~g}+$ dried coconut, $30 \mathrm{~g}$ ), (cheese, $40 \mathrm{~g}+$ boiled potatoes, $50 \mathrm{~g}$ ). The three diets were presented to group of 6 rats on day 1, 2 and 3 respectively and then repeated in same succession for 40 days. These diets were provided in addition to normal pellet chow.

\section{Experimental Design}

Following one week of acclimatization, the rats were randomly divided into 6 groups with six rats each: Group I: Normal control rats fed on Standard Chow Diet

Group II: Obesity control rats fed on High Fat Cafeteria Diet (HFCD) for 40 days

Group III: Rats fed with HFCD and treated with MEHF at a dose level of $100 \mathrm{mg} / \mathrm{kg}$ b.w. /day orally for 40 days Group IV: Rats fed with HFCD and treated with MEHF at a dose level of $200 \mathrm{mg} / \mathrm{kg}$ b.w. /day orally for 40 days Group V: Rats fed with HFCD and treated with MEHF at a dose level of $400 \mathrm{mg} / \mathrm{kg}$ b.w. /day orally for 40 days Group VI: Rats fed with HFCD and treated with Standard drug orlistat at a dose level of $50 \mathrm{mg} / \mathrm{kg}$ b.w. /day orally for 40 days

\section{Sample collection and Biochemical Analysis}

At the end of experimental period of 40days, the animals were sacrificed by cervical decapitation. The blood was collected and the serum was separated and used for various biochemical analyses. The liver, kidney, spleen and fat pads (mesenteric and perirenal fat pads) were dissected out, washed in ice cold saline, blotted dry and weighed. Liver tissues were homogenized in $0.1 \mathrm{M}$ phosphate buffer $(\mathrm{pH} 7.4)$ and the homogenate was used to analyze the antioxidant status.

In serum, the levels of glucose [9], total cholesterol [10], triglycerides [11], HDL cholesterol [12], LDL cholesterol [12], VLDL cholesterol [12] and biochemical markers of hepatic injury viz. aspartate transaminase (AST) [13], alanine transaminase (ALT) [13] and alkaline phosphatase (ALP) [13] were analyzed. Tissue lipid peroxides (LPO) [14], superoxide dismutase (SOD) [15], glutathione peroxidase (GPX) [16], reduced glutathione [17] and catalase [18] were determined in liver homogenate.

\section{Statistical Analysis}

All the data reported are expressed as mean \pm S.E.M. Statistical analysis was performed using the Student's ttest. The values were considered to be significantly different when the P-value was less than 0.05 compared to baseline or control values.

\section{Results}

\section{Effect on Body Weight Gain, Organ Weight and Fat Pad Weight}

After 40 days of HFCD administration, body weight, organ weight and fat pad (mesenteric, perirenal) weights increased significantly in high fat cafeteria diet fed obesity control group (Group II) animals as compared with the normal control group (Group I) animals which were fed with normal pellet chow. However, rats fed on HFCD when treated with MEHF (100mg, 200mg, 400mg/kg b.w.) showed a significant decrease in body weight gain, organ weight and fat pad weights in a dose dependent manner when compared with the group II animals [Table 1]. 
Table: 1 Effect of MEHF on Organ and Fat Pad Weight in HFCD induced obese rats

\begin{tabular}{|c|c|c|c|c|}
\hline Groups & Liver(g) & Spleen(g) & Mesenteric Fat Pad(g) & Perirenal Fat Pad(g) \\
\hline Group I & $5.49 \pm 1.06$ & $0.55 \pm 0.04$ & $4.95 \pm 0.49$ & $0.95 \pm 0.02$ \\
\hline Group II & $7.85+0.70$ & $0.89 \pm 0.06$ & $15.25 \pm 0.09$ & $4.35 \pm 0.05$ \\
\hline Group III & $7.46 \pm 1.09$ & $0.91+0.04$ & $13.47+0.83$ & $4.07 \pm 0.06$ \\
\hline Group IV & $7.24+1.16$ & $0.73 \pm 0.05$ & $10.22+0.71$ & $3.09 \pm 0.06$ \\
\hline Group V & $6.87 \pm 1.02$ & $0.67 \pm 0.07$ & $9.52 \pm 0.62$ & $1.95 \pm 0.04$ \\
\hline Group VI & $5.64+0.66$ & $0.54+0.03$ & $5.69+0.27$ & $1.02 \pm 0.01$ \\
\hline
\end{tabular}

Values are mean \pm S.E.M, $(\mathrm{n}=6)$

*-Compared between Normal and Disease control $(\mathrm{P} \leq 0.05)$

** -Compared between Disease Control and High Dose Drug Treated Group $(\mathrm{P} \leq 0.05)$

\section{Effect on Serum Lipid Profile}

Serum lipids, such as total cholesterol, triglycerides, LDL-cholesterol and VLDL-cholesterol were significantly elevated and HDL-cholesterol was significantly decreased in the HFCD fed obese group animals (Group II), when compared to the normal controls animals (Group I). However, rats fed with HFCD when treated with MEHF (100mg, 200mg, 400mg/kg body weight) showed a significant improvement in these changes in a dose dependent manner [Table 2].

Table: 3 Effect of MEHF on Serum Lipid Profile in HFCD induced obese rats

\begin{tabular}{|l|c|c|c|c|}
\hline Groups & Cholesterol(mg/dl) & Triglycerides(mg/dl) & HDL- Cholesterol(mg/dl) & LDL- Cholesterol(mg/dl) \\
\hline Group I & $119.65 \pm 4.56$ & $93.96 \pm 3.27$ & $45.97 \pm 4.83$ & $74.83 \pm 2.84$ \\
\hline Group II & $264.60 \pm 7.87$ & $218.50 \pm 12.33$ & $27.99 \pm 3.64$ & $91.72 \pm 2.25$ \\
\hline Group III & $205.58 \pm 4.56$ & $159.18 \pm 2.78$ & $30.67 \pm 3.86$ & $90.48 \pm 2.34$ \\
\hline Group IV & $192.28 \pm 4.19$ & $122.45 \pm 2.57$ & $34.71 \pm 3.54$ & $85.55 \pm 2.71$ \\
\hline Group V & $119.75 \pm 4.42$ & $99.69 \pm 2.98$ & $42.57 \pm 3.37$ & $77.27 \pm 2.45$ \\
\hline Group VI & $103.23 \pm 2.67$ & $85.93 \pm 0.91$ & $44.62 \pm 0.75$ & $39.67 \pm 0.76$ \\
\hline
\end{tabular}

\section{Effect on Blood Glucose and Serum Hepatic Marker Enzymes}

The concentration of blood glucose was higher in HFCD fed obese control rats (Group II) than in rats fed with normal diet (Group I). Whereas, MEHF (100mg, 200mg, 400mg/kg b.w.) supplementation normalized the blood glucose level [Table 3] significantly. Also, the data of the present study showed significant increase in the activities of hepatic enzymes such as aspartate transaminase (AST), alanine transaminase (ALT) and alkaline phosphatase (ALP) in HFCD fed obese control rats (Group II) when compared to group I animals fed with normal rat chow pellet whereas, the levels of AST, ALT and ALP significantly reduced in association with MEHF treatment [Table 3].

Table: 3 Effect of MEHF on Blood glucose and Hepatic Markers level in HFCD induced obese rats

\begin{tabular}{|l|l|l|l|l|}
\hline Groups & Glucose(mg/dl) & AST(U/L) & ALT(U/L) & ALP(U/L) \\
\hline Group I & $87.12 \pm 1.24$ & $65.76 \pm 0.80$ & $38.27 \pm 0.91$ & $78.25 \pm 0.74$ \\
\hline Group II & $296.56 \pm 4.87^{\mathrm{a}^{*}}$ & $160.03 \pm 0.89$ & $110.46 \pm 1.27$ & $146.27 \pm 1.59$ \\
\hline Group III & $244.98 \pm 2.34$ & $145.67 \pm 1.66$ & $89.42 \pm 1.17$ & $130.86 \pm 1.45$ \\
\hline Group IV & $201.32 \pm 2.98$ & $111.54 \pm 1.35$ & $71.36 \pm 1.23$ & $108.21 \pm 1.32$ \\
\hline Group V & $153.87 \pm 2.87^{\mathrm{b}^{*}}$ & $90.51 \pm 1.39$ & $54.18 \pm 0.81$ & $91.33 \pm 0.90$ \\
\hline Group VI & $89.21 \pm 0.87^{\mathrm{c}^{*}}$ & $70.12 \pm 1.67$ & $39.53 \pm 0.92$ & $80.12 \pm 1.07$ \\
\hline
\end{tabular}

\section{Effects on Hepatic Antioxidant Enzymes and Lipid Peroxidation}

The effects of MEHF on hepatic antioxidant enzyme activities are presented in Table 4. The Group-II animals administered with HFCD resulted in the decreased level of reduced glutathione, glutathione peroxidase, superoxide dismutase and catalase and increased rate of lipid peroxidation when compared to rats fed normal pellet chow (Group I). Treatment with different dose levels (100, 200, 300 mg $/ \mathrm{kg}$ b.w.) of MEHF resulted in increased levels of reduced glutathione peroxidase, Superoxide dismutase and catalase. In the respect of lipid peroxidation in liver, treatment with MEHF resulted in significant reduction in the rate of lipid peroxidation [Table 4].

Table: 4 Effect of MEHF on Antioxidant enzyme level in HFCD induced obese rats

\begin{tabular}{|l|l|l|l|l|l|}
\hline Groups & \multicolumn{1}{|c|}{ MDA } & GSH & GP $_{\mathbf{x}}$ & SOD & \multicolumn{1}{c|}{ CAT } \\
\hline Group I & $1.22 \pm 0.06$ & $4.82 \pm 0.23$ & $4.58 \pm 0.21$ & $2.66 \pm 0.01$ & $15.21 \pm 0.07$ \\
\hline Group II & $5.85 \pm 0.32$ & $1.42 \pm 0.16$ & $0.36 \pm 0.02$ & $0.45 \pm 0.02$ & $2.76 \pm 0.04$ \\
\hline Group III & $3.15 \pm 0.17$ & $1.72 \pm 0.31$ & $1.87 \pm 0.90$ & $0.97 \pm 0.02$ & $3.92 \pm 0.05$ \\
\hline Group IV & $2.42 \pm 0.15$ & $1.93 \pm 0.14$ & $1.04 \pm 0.47$ & $1.04 \pm 0.03$ & $4.35 \pm 0.03$ \\
\hline Group V & $1.91 \pm 0.13$ & $2.43 \pm 0.03$ & $0.54 \pm 0.02$ & $1.12 \pm 0.02$ & $6.76 \pm 0.03$ \\
\hline Group VI & $1.97 \pm 0.12$ & $4.58 \pm 0.21$ & $1.70 \pm 0.09$ & $2.13 \pm 0.02$ & $13.50 \pm 0.24$ \\
\hline
\end{tabular}




\section{Discussion}

It is observed that the global prevalence of obesity is increasing rapidly among adults as well as among children and is associated with serious mortalities including a high incidence of type 2 diabetes, hyperlipidemia, hypercholesterolemia, fatty liver, cardiovascular diseases, osteoarthritis as well as an increased risk of many forms of cancer [19]. Obesity is a medical condition in which excess body fat has been accumulated mainly due to sedentary life styles, lack of exercise and intake of energy rich high fat diet. The currently available treatment options are not potent enough to control obesity permanently besides they produce side effects. Hence, there is a great demand for safer and long term effective drugs to treat this global epidemic problem. Medicinal herbs are indispensable parts of traditional medicines and there is a big renaissance of the herbal medicines globally as these drugs are effective and safe without any side effects. Hence, in the present work the anti-obesity potential of MEHF in rats fed a high fat cafeteria diet (HFCD) was investigated by analyzing the body weight, organ and fat pad weight and blood and tissue biochemical profiles.

The gain in body weight, organ and fat pad weights were significantly increased in HFCD fed rats when compared with the normal diet fed rats. This increase in weight might be due to increased energy intake leading to increased fat deposition in tissue and organs. While HFCD fed rats when treated with MEHF, the gain in body weight, organ and fat pad weights significantly decreased. It was also observed that administration of MEHF did not alter food intake in treated rats, indicating that the prevention of weight gain induced by this extract was not due to a reduction of energy intake. MEHF might have increased the catabolism of lipids in adipose tissue resulting in a decrease in mean body weight.

The elevation in serum concentrations of Total Cholesterol (TC), Low Density Lipoprotein Cholesterol (LDL-C), Very Low Density Lipoprotein Cholesterol (VLDL-C), Triglycerides (TG) along with decreased concentration of High Density Lipoprotein Cholesterol (HDL-C) observed in HFCD fed disease control rats are the major risk factors for the development of coronary heart disease and atherosclerosis. The significant increase in TC and TG level in cafeteria diet fed animals can be attributed to increase in both de novo synthesis and intestinal absorption of cholesterol [20]. Also, increased oxidative stress produces reactive oxygen species (ROS) which react with lipoproteins to produce oxidation states, thus diminishing the cellular uptake of lipids from the blood [21, 22]. Data of this study suggested that a 40 days administration of MEHF exerts a positive effect on lipid profile i.e, decreased the serum concentration of TC, LDL-C, VLDL-C, TG and increased the concentration HDL-C in treated group animals when compared with untreated disease control group. The results may be partly caused by the decreased absorption of cholesterol from the diet or by the antioxidants present in the plant drug might have contributed to elevated cellular lipid uptake [23]. Also, a substantial reduction of total cholesterol in serum in the plant drug treated groups could be attributed to a reduction in the activities of the liver enzyme 3-hydroxy-3-methyl glutaryl coenzyme A (HMG-CoA) reductase, which is a ratelimiting enzyme in cholesterol biosynthesis. A higher content of HDL-C is correlated with a reduced risk of coronary heart disease. The increased level of HDL-C facilitates the transport of cholesterol from the serum to the liver, where it is catabolized and excreted. The decrease of TG in plant drug treated groups may be attributed to an increase in the activity of endothelium bound lipoprotein lipase that hydrolyses the triglycerides.

The significant increase in glucose in cafeteria diet fed animal can be due to defective insulin synthesis and decreased insulin efficiency [24]. Treatment with MEHF almost normalized the glucose levels in dose dependent manner. The test drug might have enhanced the secretion of insulin from the $\beta$-cells of the islets of pancreas or increased the efficiency of insulin which facilitates the delivery of glucose from blood to target tissues.

The high levels of serum enzymes (AST, ALT and ALP) in group II animals are attributed to fatty liver induced by a HFCD. Fatty liver associated with obesity is an independent risk factor for liver peroxidation which results in cell damage and elevation of the levels of these enzymes in serum. AST, ALT and ALP were significantly lowered in rats administered with MEHF, suggesting the amelioration of fatty liver. This decrease may be due to the consequence of prevention of liver damage by the antioxidant potential of the extract [ 25 , 26].

Generally obesity is associated with oxidative stress which results from an imbalance between the production of free radicals and an effective antioxidant system. Reduced glutathione (GSH) constitutes the first line of defense against free radicals in the liver, and it is also responsible for the maintenance of protein thiols and act as a substrate for Glutathione peroxidase (GPx). The results indicated that GSH contents depleted in the rats with obesity induced by a high fat diet, and were restored after the treatment with MEHF. Enzymatic antioxidants, like superoxide dismutase (SOD), catalase (CAT) or GPx, can scavenge reactive oxygen species (ROS) and free radicals or prevent their formation. The present study suggested decreased activities of antioxidant enzymes SOD, CAT and GPx in the liver of rats fed with high fat cafeteria diet as compared to those on normal diet and these results are in agreement with reports of earlier workers which suggest that feeding a high fat diet to experimental animals depresses their antioxidant system due to increased lipid peroxidation and formation of free radicals [27]. On treatment with MEHF the activities of antioxidant enzymes 
(SOD, CAT and GPx) were significantly elevated in liver and this enhanced antioxidant capacity might have been mediated through natural antioxidants present in the plant drug.

\section{Conclusions}

It can be concluded from the data that, administration of a methanolic extract of leaves of Heritiera fomes regulates serum lipid profiles, reduces the oxidative stress and decreases adipose tissue mass and body weight gain. The mechanism induced by methanolic extract of Heritiera fomes to be further researched.

\section{References}

[1]. Vinay Kumar, Uma Bhandari, Chakra Dar Tripathi, Geetika Khanna. Evaluation of antiobesity and cardioprotective effect of Gymnema sylvestre extract in murine model. Indian J Pharmacol 2012; 44(5): 607-613.

[2]. Mokdad A, Ford E, Bowman B, Dietz W, Vinicor F, Bales V, Marks J. Prevalence of obesity, diabetes, and obesity related health risk factors. JAMA 2003; 289: 76-79.

[3]. Cragg GM, Newman DJ. Natural Product Drug Discovery in the Next Millennium. Pharm Biol 2001; 39(1): 8-17.

[4]. Ali, K. Nahar, M. Sintaha et al., "An evaluation of antihyperglycemic and antinociceptive effectsof methanol extract of Heritiera fomes Buch- Ham. (Sterculiaceae) barks in Swiss albino mice,” Advances in Natural and Applied Sciences, vol. 5, no. 2, pp. 116$121,2011$.

[5]. M. A. Hossain, S. Panthi, M. Asadujjaman, S. R. Khan, F. Ferdous, and S. K. Sadhu, "Phytochemical and pharmacological assessment of the ethanol leaves extract of Heritiera fomes Buch. Ham. (Family- Sterculiaceae)," Journal of Porphyrins and Phthalocyanines, vol. 2, pp. 95-101, 2013.

[6]. Wangensteen H, Dang HC, Uddin SJ, Alamgir M, Malterud KE. Antioxidant and antimicrobial effects of the mangrove tree Heritiera fomes. Nat Prod Commun. 2009; 4(3):371-6.

[7]. J. K. Patra and H. Thatoi, "Anticancer activity and chromatography characterization of methanol extract of Heritiera fomes Buch. Ham., a mangrove plant from Bhitarkanika, India," Oriental Pharmacy and Experimental Medicine, vol. 13, no. 2, pp. 133-142, 2013.

[8]. Harris RB. The impact of high- or low-fat cafeteria foods on nutrient intake and growth of rats consuming a diet containing $30 \%$ energy as fat. Int J Obes 1993; 17: 307-315.

[9]. Folin O and Wu H. A system of blood analysis. J Biol Chem 1919; 38: 81-110.

[10]. Parekh AC, Jung DH. "Determination of Cholesterol with ferric acetate, Uranium Acetate and sulphuric acid-ferrous sulphate reagents". Anal Chem 1970; 42: 1523-1529.

[11]. Foster LB, Dunn RT. "Stable reagents for determination of Serum triglycerides by a colorimetric Hantzsch condensation method". Clin Chem 1973; 196: 338-340.

[12]. Friedewald WT, Levy RI, Fredrickson DS. Estimation of the concentration of low density lipoprotein cholesterol in plasma without the use of preparative ultracentrifuge. J Clin Chem 1972; 18(6): 499-502.

[13]. King J. In: Practical Clinical Enzymology, Princeton MJ (Fol) Van D Nostrand Company, and London 1965: 363.

[14]. Ohkawa H, Ohishi N, Yagi K. "Assay of lipid peroxides in animal tissues for thiobarbituric acid reaction". Annual Biochem 1979; 95: $351-358$

[15]. Misra HP, Fridovich I. "The role of superoxide anion in the autooxidation of epinephrine and a simple assay for SOD". J Biol Chem 1972; 247: 3170-3175.

[16]. Rotruck JT, Pope AC, Ganther H, Swanson AB, Hafeman DG, Hoeksirawa. "Selenium: Biochemical role as a component of Glutathione Peroxidase". Science 1973; 179(73), 588-590.

[17]. Moron MS, Depierre JW, Mannervik B. "Levels of glutathione, glutathione reductase and glutathione-S-transferase activities in rat lung and liver". Biochim and Biophys Acta 1979; 5820: 60-68.

[18]. Sinha AK. "Calorimetric assay of catalase". J Biochem 1972; 47:389-394.

[19]. Yilmaz A, Suleyman H, Umudum Z, Sahin YN. The effect of adrenalectomy on leptin levels and some metabolic parameters in rats with diet-induced obesity. Biol Pharm Bull 2002; 25: 580—583.

[20]. Jiao S, Matsuzawa Y, Matsubara K, Kubo M, Tokunaga K. Abnormalities of plasma lipoproteins in a new genetically obese rat with non-insulin dependent diabetes mellitus (Wistar fatty rat). Int J Obes 1991; 15: 487-495.

[21]. Diniz YS, Rocha KKHR, Souza GA, et al. Effects of N-acetylcysteine on sucrose-rich diet-induced hyperglycaemia dyslipidemia and oxidative stress in rats. Eur J Pharmacol 2006; 543: 151-157.

[22]. Brizzi P, Tonolo G, Carusillo F, Malaguarnera M, Maioli M, Musumeci S. Plasma lipid composition and LDL oxidation. Clin Chem Lab Med 2003; 41: 56-60.

[23]. Irene PT, Ilias PD, Laskarina MK, George A, Ioannis SV, Alkisti P et al. Water Soluble Vitamin E Administration in Wistar Rats with Non-alcoholic Fatty Liver Disease. The Open Cardiovascular Medicine Journal 2012; 6: 88-97.

[24]. Kahn BB, Flier JS. Obesity and insulin resistance. J Clin Invest 2000; 106: 473-481.

[25]. Zamora R, Hidalgo FJ, Tappel AL. Comparative antioxidant effectiveness of dietary b-carotene, vitamin E, selenium and coenzyme Q10 in rat erythrocytes and plasma. J Nutr 1991; 121: 50-56.

[26]. Nakano T, Kanmuri T, Sato M, Takeuchi M. Effect of astaxanthin rich red yeast (Phaffa rhodozyma) on oxidative stress in rainbow trout. Biochim Biophys Acta 1999; 1426: 119-125.

[27]. Moonkyu K, Jung WO, Hee KL, Hwan SC, Sang ML, Changsook K et al. Anti-obesity Effect of PM-F2-OB, an Anti-obesity Herbal Formulation, on Rats Fed a High-Fat Diet. Biol Pharm Bull 2004; 27(8): 1251-1256.

IOSR Journal of Pharmacy and Biological Sciences (IOSR-JPBS) is UGC approved Journal with Sl. No. 5012, Journal no. 49063.

M.Salim Mirza. "Anti-Obesity Potential of Heritiera Fomes Leaves Methanolic Extract in a Preclinical Model." IOSR Journal of Pharmacy and Biological Sciences (IOSR-JPBS) 12.4 (2017): 01-05. 